\section{Insulin promoter factor-1}

K. J. Lackner ${ }^{1}$ und D. Peetz ${ }^{2}$

${ }^{1}$ Institut für Klinische Chemie und Laboratoriumsmedizin, Universitätsmedizin Mainz, Mainz, Deutschland

${ }^{2}$ Institut für Labormedizin, Helios Klinikum Berlin-Buch, Berlin, Deutschland

Synonym(e) Glukosesensitiver Faktor; IPF-1

Englischer Begriff insulin promoter factor-1

Definition Homeobox-Domain-Transkriptionsfaktor, der die Insulin- und Somatostatinexpression in Pankreas und Duodenum reguliert. Daneben relevant für die Pankreasentwicklung.
Molmasse $30,8 \mathrm{kDa}$.

Beschreibung Defekte von IPF-1 sind eine seltene Ursache des ,maturity onset diabetes of the young“ (MODY), der auch als Typ-IV-MODY bezeichnet wird. Die Defekte gehen mit einer gestörten Regulation der Genexpression bei der Entwicklung von pankreatischen $\beta$-Zellen und deren Funktion einher.

\section{Literatur}

Fajans SS, Bell GI, Polonsky KS (2001) Molecular mechanisms and clinical pathophysiology of maturity-onset diabetes of the young. N Engl J Med 345:971-980 